\title{
Basaloid squamous cell carcinoma of the oral cavity: A case series
}

\author{
Kenji Yamagata, Rei Karube, Toru Yanagawa, Wolfgang Zemann, \\ Philipp Metzler, Kojiro Onizawa, Hiroki Bukawa
}

\begin{abstract}
Introduction: Basaloid squamous cell carcinoma (BSCC) has been reported as a highly aggressive, malignant tumor with a high rate of both local recurrence and regional and distant metastases. The purpose of this review is to examine the characteristics and prognosis of BSCC occurring in the oral cavity. Case Series: We here present three cases of oral BSCC. Case 1: A 49-year-old male was clinically and radiologically classified as T3NoMo (Stage III).
\end{abstract}

Kenji Yamagata ${ }^{1}$, Rei Karube ${ }^{2}$, Toru Yanagawa ${ }^{3}$, Wolfgang Zemann ${ }^{4}$, Philipp Metzler ${ }^{4}$, Kojiro Onizawa ${ }^{5}$, Hiroki Bukawa $^{6}$

Affiliations: ${ }^{1}$ DDS, Ph.D, Assistant Professor, Department of Oral and Maxillofacial Surgery, Clinical medicine, Faculty of Medicine, University of Tsukuba, Tsukuba, Japan; ${ }^{2}$ DDS, Researcher, Department of Oral and Maxillofacial Surgery, Clinical medicine, Faculty of Medicine, University of Tsukuba, Tsukuba, Japan; ${ }^{3} \mathrm{MD}$, DDS, PhD, Associate Professor, Department of Oral and Maxillofacial Surgery, Clinical medicine, Faculty of Medicine, University of Tsukuba, Tsukuba, Japan; ${ }^{4} \mathrm{MD}$, DDS, Consultant, Department of Cranio-Maxillofacial and Oral Surgery, University of Zurich, Zurich, Switzerland; ${ }^{5}$ DDS, Ph.D, Professor, Department of Oral and Maxillofacial Surgery, Clinical medicine, Faculty of Medicine, University of Tsukuba, Tsukuba, Japan; ${ }^{6} \mathrm{MD}$, DDS, Ph.D, Professor, Department of Oral and Maxillofacial Surgery, Clinical medicine, Faculty of Medicine, University of Tsukuba, Tsukuba, Japan.

Corresponding Author: Kenji Yamagata, Department of Oral and Maxillofacial Surgery, Clinical Medicine, Faculty of Medicine, University of Tsukuba, 1-1-1 Tennodai, Tsukuba city, Ibaraki, 305-8575, Japan; Tel: +81-29-8533052; Fax: +81-29-853-3052; Email: ykenji@md.tsukuba.ac.jp

Received: 02 June 2012

Accepted: 17 September 2012

Published: 01 April 2013
He underwent chemoradiotherapy and more than 8 years after treatment, the patient is alive without evidence of recurrence. Case 2: A 69year-old female was classified as T4aNoMo (Stage IVA). She underwent chemoradiotherapy, and died from lung and liver metastasis 4 years and 3 months after her first visit. Case 3: A 73year-old male was clinically and radiologically classified as T2N2cMo (Stage IVA). The patient underwent chemoradiotherapy and bilateral neck dissection. More than about 5 years after surgery, the patient is alive without evidence of recurrence. Discussion: About a hundred cases of oral BSCC have been reported, and we review previous reports of oral BSCC cases. Oral BSCC has a lower rate of distant metastasis than head and neck BSCC. Conclusion: In contrast to the aggressive behavior of BSCC occurring in the head and neck, BSCC occurring in the oral cavity appears to have a lower rate of distant metastasis than head and neck BSCC. When it comes to the follow-up of patients for metastases, a more thorough work-up is recommended for patients with oral BSCC than for patients with SCC.

Keywords: Basaloid squamous cell carcinoma (BSCC), Oral, Cervical lymph node metastasis, Distant metastasis

$$
* * * * * * * * *
$$

Yamagata K, Karube R, Yanagawa T, Zemann W, Metzler P, Onizawa K, Bukawa H. Basaloid squamous cell carcinoma of the oral cavity: A case series. International Journal of Case Reports and Images 2013;4(4):199-207.

$$
* * * * * * * * *
$$

doi:10.5348/ijcri-2013-04-294-CS-3 


\section{INTRODUCTION}

Basaloid squamous cell carcinoma (BSCC), first described in the head and neck by Wain et al. in 1986 [1], is a rare subtype of squamous cell carcinoma (SCC). In the upper aerodigestive tract, BSCC occurs most frequently at the base of the tongue, the larynx, and the hypopharynx [1-3]. The neoplasma is composed chiefly of basaloid cells with typical foci of squamous differentiation, and the basaloid cells have dark hyperchromatic nuclei and scant cytoplasm. The neoplasma occasionally shows peripheral cellular palisading, comedonecrosis, and intratumoral cystic spaces [4].

Head and neck BSCC, which is considered to be a highly aggressive malignant tumor, is characterized by a high rate of both local recurrence and regional and distant metastases $[2,3,5]$. Recently, some studies have reported relatively large numbers of patients who presented with head and neck BSCC, and have attempted to determine whether their outcomes differed from the outcomes of patients with similar presentations of common SCC [6, 7].

In the oral cavity, BSCC has a predilection for the tongue [2, 4, 8], although it has been described in other locations including the floor of the mouth $[2,9]$, the palate $[5,10]$, the buccal mucosa [11] and the gingiva [12]. The prognosis of oral BSCC is reported to be worse than that of conventional SCC due to the advanced stage at which cases have been discovered [13]. However, it has also been reported that the prognosis of oral BSCC does not differ from that of conventional SCC [8]. About a hundred cases of oral BSCC have been reported, but almost all of the sample sizes have been too small to summarize its behavior and prognosis [2, 4, 5, 8-18]. Since the incidence of oral BSCC is low, studies encompassing oral BSCC have often included cases of BSCC originating in the head and neck, which precludes reliable findings on the clinical behavior of oral BSCC specifically. In this article, we report three cases of oral BSCC and review previous reports of oral BSCC cases.

\section{CASE SERIES}

Case 1: A 49-year-old Japanese male was referred to the Department of Oral and Maxillofacial Surgery at Tsukuba University Hospital with a 3-month history of a mass lesion in his left soft palate. He had a medical history of gout. Examination of the oral cavity revealed a red, irregular granulation-like mass in the soft palate towards the retromandibular region (Figure 1). The regional lymph nodes were normal. A chest X-ray revealed no lung metastasis. An incisional biopsy performed under local anesthesia was histopathologically diagnosed as BSCC. Microscopic examination showed epithelial-like and basaloid tumor cells with an alveolar architecture and peripheral palisading, hyperchromatic nuclei with a high nuclear/cytoplasmic (N/C) ratio, and frequent mitosis (Figure 2). The patient was clinically and radiologically

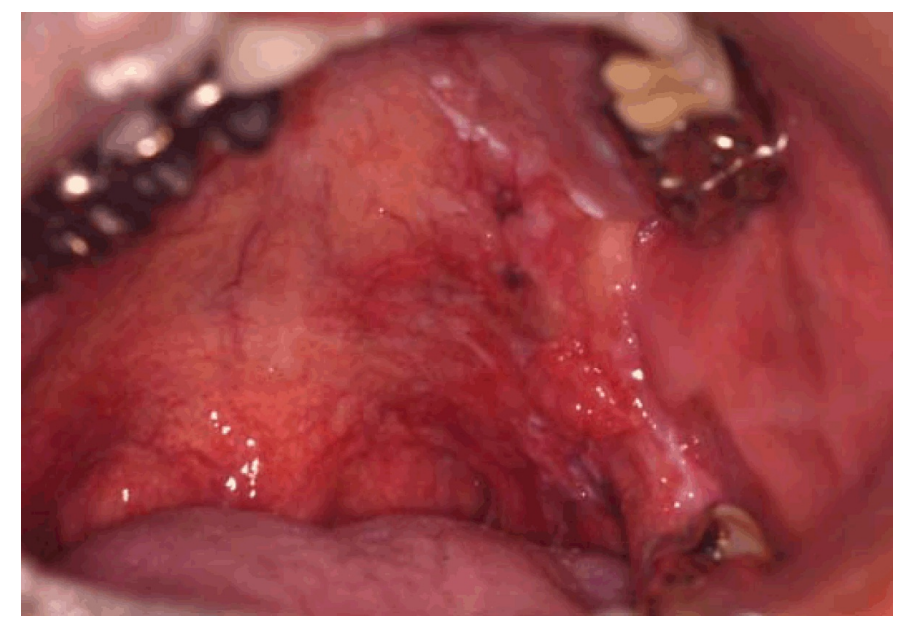

Figure 1: Intra-oral tumor appearance (Case 1). Examination of the oral cavity revealed a red, irregular, granulation-like mass in the soft palate towards the retromandibular space.
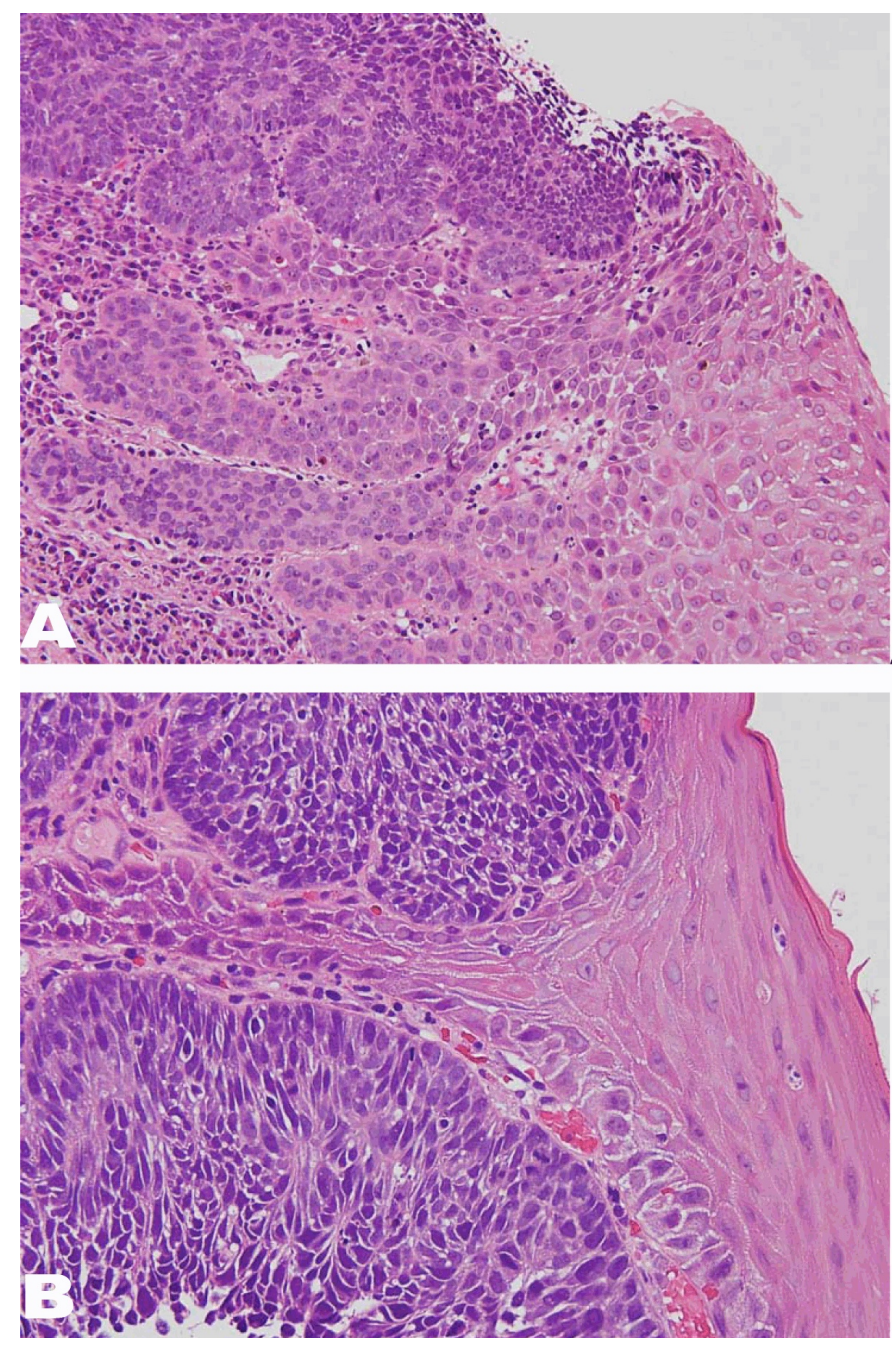

Figure 2 (A and B): Pathological appearance (Case 1). Microscopic examination showed alveolar architecture and peripheral palisading formed by epithelial-like and basaloid tumor cells. These cells showed hyperchromatic nuclei with a high N/C ratio and frequent mitosis. Magnification: (A) x100, (B) $\mathrm{x} 400$. 
classified as T3NoMo (Stage III). The patient underwent chemoradiotherapy with a total delivered dose of 66.6 Gy along with 5-fluorouracil and cisplatin or nedaplatin. More than eight years after radiotherapy, the patient is alive without evidence of recurrence.

Case 2: A 69-year-old Japanese female was referred to the Department of Oral and Maxillofacial Surgery at Tsukuba University Hospital with a 4-month history of difficulty of moving her tongue. Her medical history included gallstone disease, nephrosclerosis, and cataract. Examination of the oral cavity revealed a $25-\mathrm{mm}$ diameter mass in the right side of the dorsum towards the root of the tongue (Figure 3). The regional lymph nodes were normal. A chest X-ray revealed no lung metastasis. An incisional biopsy was performed under local anesthesia. Although microscopic examination showed partial adenoid cystic differentiation, epitheliallike and basaloid tumor cells had formed an alveolar and palisading architecture, and the tissue was histopathologically diagnosed as BSCC (Figure 4). The patient was clinically and radiologically classified as T4aNoMo (Stage IVA). She refused radical surgery and underwent chemoradiotherapy with a total delivered Xray dose of 52.2 Gy and proton beam dose of $27.6 \mathrm{~Gy}$, along with 5-fluorouracil and nedaplatin. This met with only a partial clinical response, but she refused more radical surgery. Three and a half years later, both regrowth of the primary tumor and metastasis to the lung and liver were observed. The patient died from lung and liver metastasis 4 years and 3 months after her first visit.

Case 3: A 73-year-old Japanese male was referred to the Department of Oral and Maxillofacial Surgery at Tsukuba University Hospital with a 3-month history of mass lesion in the left floor of his mouth. He had a medical history of hypertension and bronchial asthma. Examination of the oral cavity revealed an irregular granulation-like 22x15 mm mass in the floor of the mouth (Figure 5). Both computed tomography (CT) scan

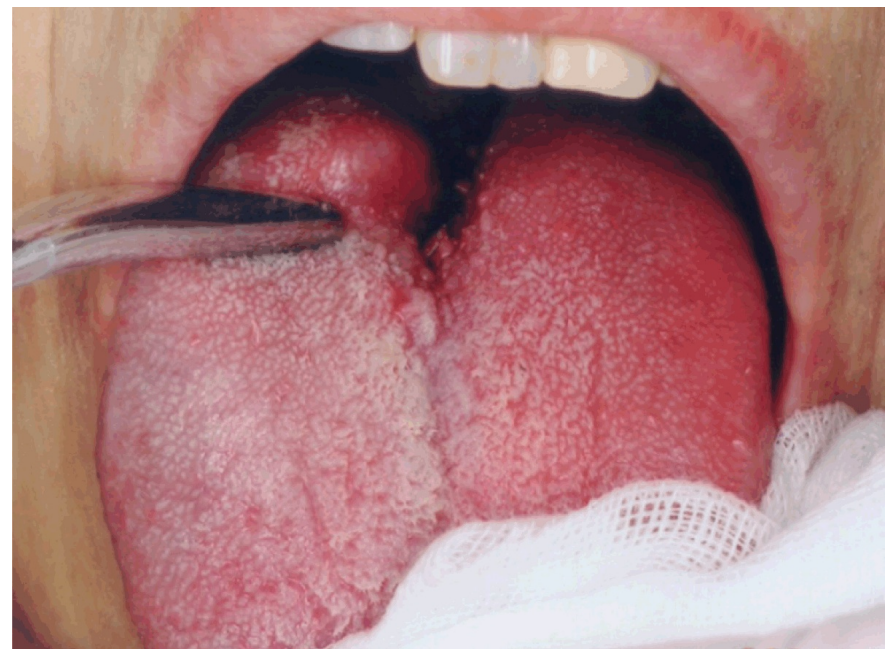

Figure 3: Intra-oral tumor appearance (Case 2). Examination of the oral cavity revealed a $25-\mathrm{mm}$ diameter mass in the right side of the dorsum towards the root of the tongue.
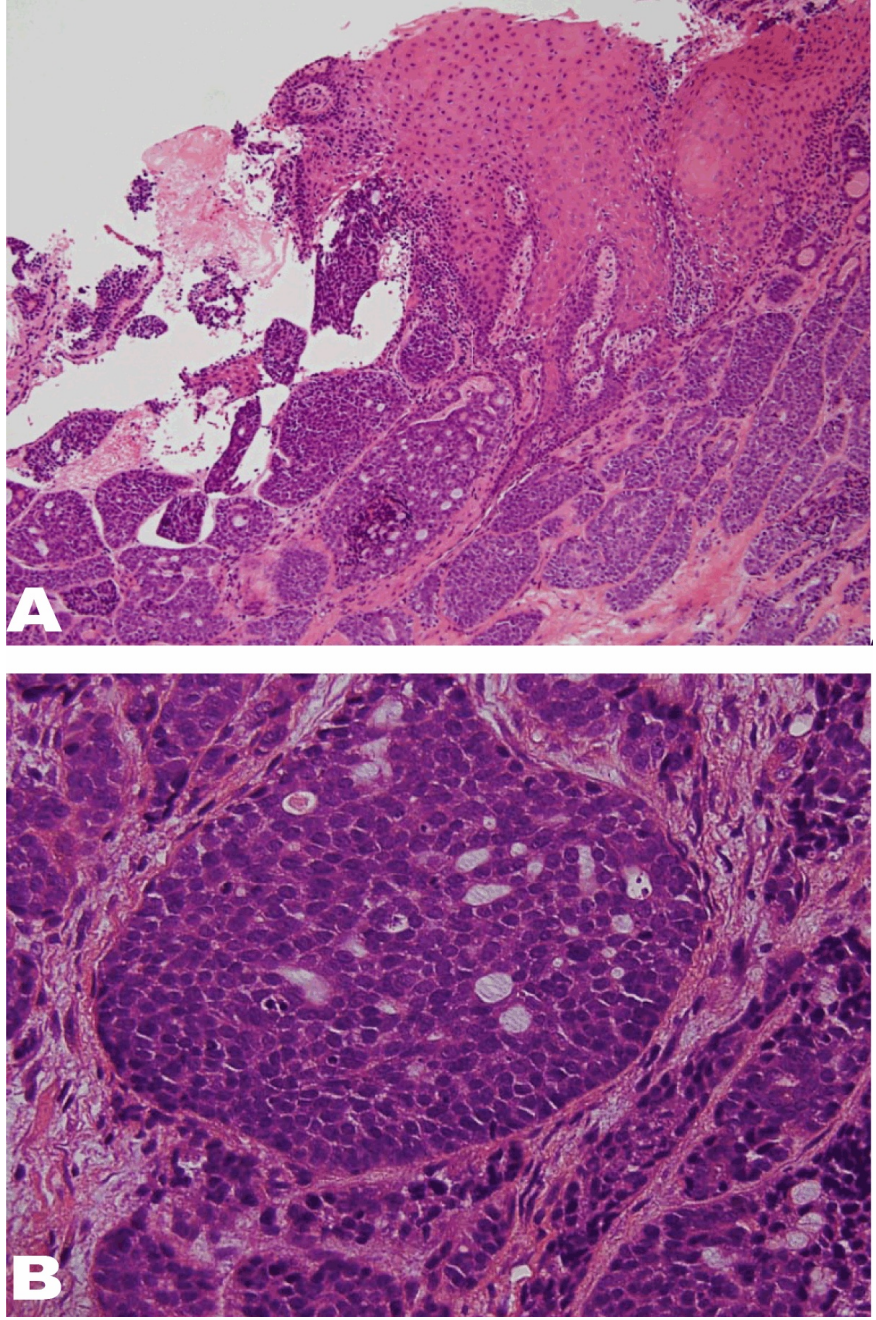

Figure 4 (A and B): Pathological appearance (Case 2). Although partial A and B adenoid cystic differentiation was present, epithelial-like and basaloid tumor cells formed palisading and alveolar architecture. Magnification: (A) x100, (B) $\mathrm{x} 400$.

and magnetic resonance imaging (MRI) scan showed swelling of the submandibular lymph nodes on both sides. Lung metastasis was not detected in the chest X-ray. An incisional biopsy performed under local anesthesia was histopathologically diagnosed as BSCC (Figure 6). Microscopic examination showed epitheliallike and basaloid tumor cells forming a lobulate or papillary alveolar architecture. These cells showed peripheral palisading along with hyperchromatic, high $\mathrm{N} / \mathrm{C}$ ratio nuclei and frequent mitosis. The patient was clinically and radiologically classified as T2N2cMo (Stage IVA). The patient underwent chemoradiotherapy with a delivered total dose of 69 Gy along with 5fluorouracil, docetaxel, and nedaplatin. The primary response was complete, but with remaining lymphadenopathy. A bilateral neck dissection was performed. More than about 5 years after surgery, the patient is alive without evidence of recurrence. 


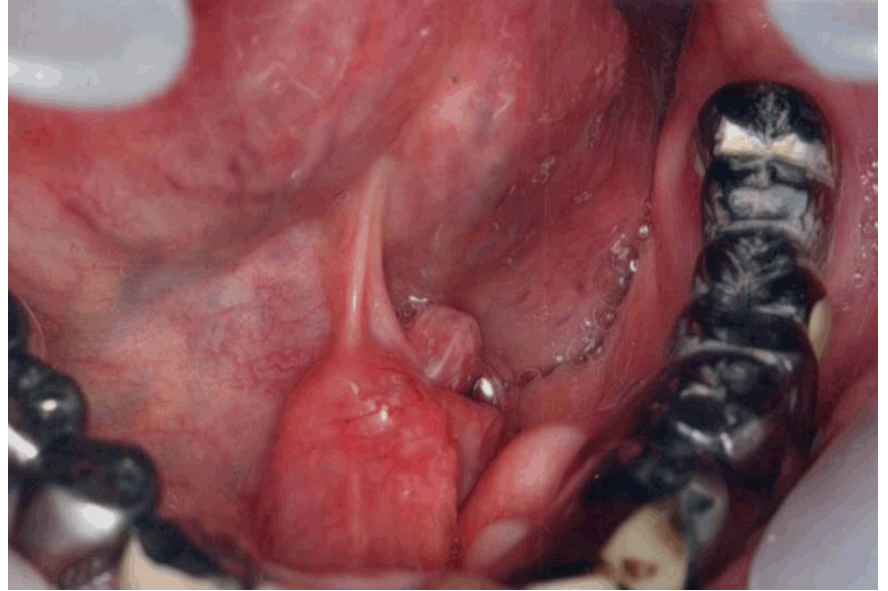

Figure 5: Intra-oral tumor appearance (Case 3). Examination of the oral cavity revealed a granulation-like, irregular, 22x15 mm mass in the floor of the mouth.
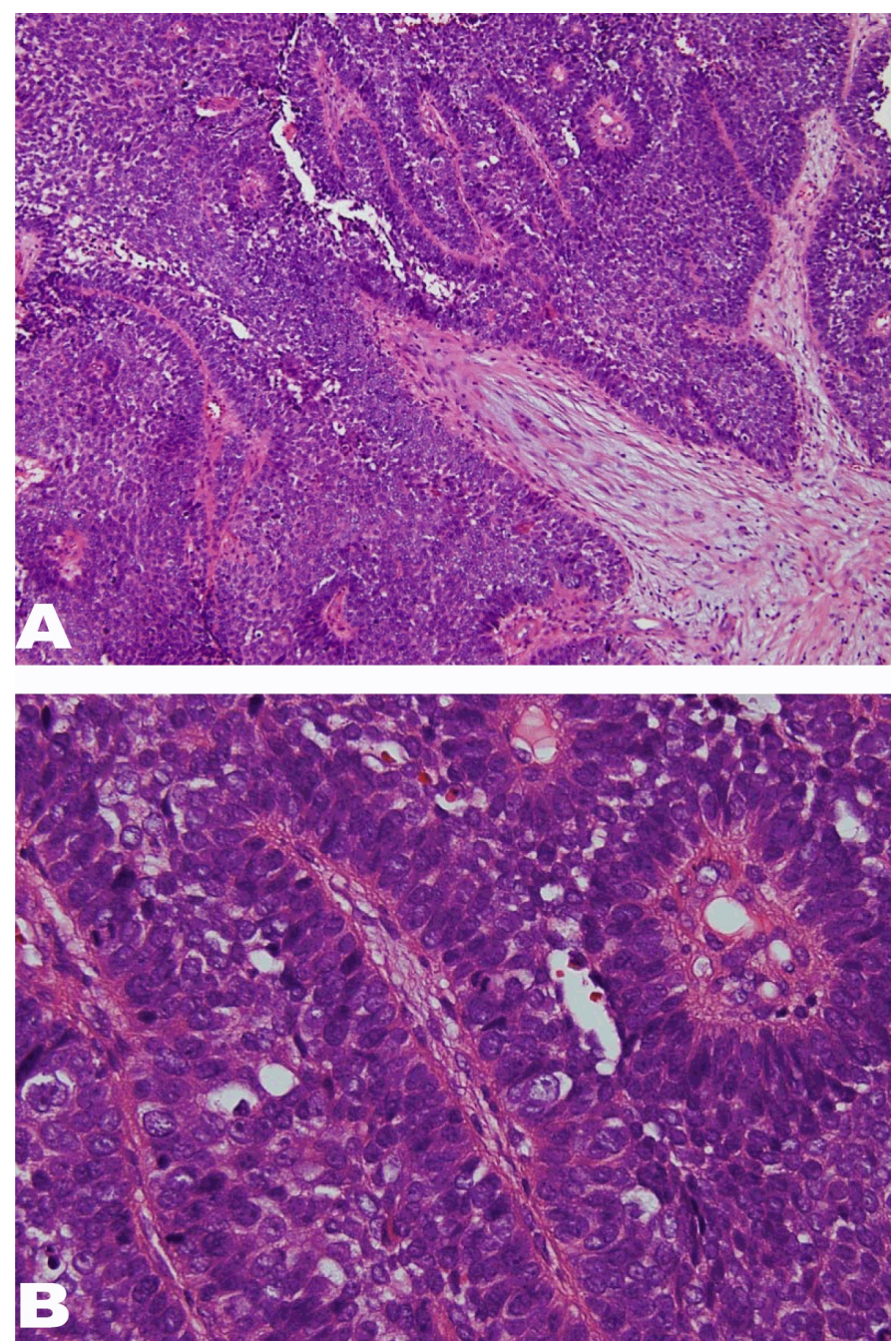

Figure 6 (A and B): Pathological appearance (Case 3). Lobulate or papillary alveolar forms were constructed by epithelial-like and basaloid tumor cells. These cells showed peripheral palisading, hyperchromatic nuclei with a high N/C ratio, and frequent mitosis. Magnification: (A) x100, (B) x400.

\section{DISCUSSION}

The BSCC has been described mostly among men in their sixth to seventh decade, and has been associated with tobacco and alcohol abuse. Most instances of BSCC present at a high tumor stage and seem to have a propensity for lymph-node and systemic metastases [7, 19]. These reports agree with our summary of previously reported oral cases, in which $72.5 \%$ of the patients were male and $27.5 \%$ were female, and the mean age was 61.4 years. Over $60 \%$ presented with a tumor stage greater than III (Tables 1 and 2).

Macroscopically, BSCCs are typically firm-to-hard exophytic nodular masses with central necrosis. The BSCC cells are referred to as basaloid because of their immature appearance, and they resemble cells of the basal layer of typical stratified squamous epithelium [19]. BSCC has two distinct phenotypes: basaloid and squamous. The most common BSCC growth pattern is solid nests with a typical cell population, basaloid at the periphery and squamous at the center.

In the oral cavity, the differential diagnosis of BSCC principally includes adenoid cystic carcinoma, polymorphous low grade adenocarcinoma (PLGA), basal cell adenocarcinoma (BCA), salivary duct carcinoma (SDC), and adenosquamous carcinoma (ASC) [13]. BSCC can be mistaken for solid ACC due to pseudogland formations with hyalinized and eosinophilic deposits. However, ACC is not associated with SCC. The cytologic and histomorphologic characteristics of solid-type ACC are quite similar to those of BSCC. Short-term ACC biologic behavior is less aggressive than that of BSCC; regional lymph node metastases are infrequent at the initial presentation, and distant metastases develop late in the follow-up [6]. Ide et al. reported the following helpful features in differentiating BSCC from solid ACC:

(1) classic cribriform patterns are limited, when present, in BSCC

(2) even in the solid type of ACC, well-formed tubular structures are almost always present, while nuclear pleomorphism, mitoses, and necrosis are rare

(3) focal squamous differentiation in the basaloid nests is only rarely evident in ACC, and most importantly

(4) ACC does not contain SCC foci or exhibit carcinomatous changes in the surface epithelium[13].

Many authors have reported that head and neck BSCC is more aggressive than SCC, whereas others have found similar prognoses for BSCC and SCC at similar stages. Soriano's study showed fewer locoregional failure-related deaths for BSCC, while the rates for distant metastases and overall survival were worse. The distant metastasis rates were $41 \%$ and $6 \%$ for BSCC and SCC, respectively [3]. Therefore, the authors recommended routinely performing a chest CT scan and FDG-PET to rule out early distant metastasis, and the inclusion of adjuvant chemotherapy in the treatment protocol. In that study, the treatment opinions were based on tumor site and stage and patient characteristics, and not on the histological subtypes currently used in routine practice. In most case studies 


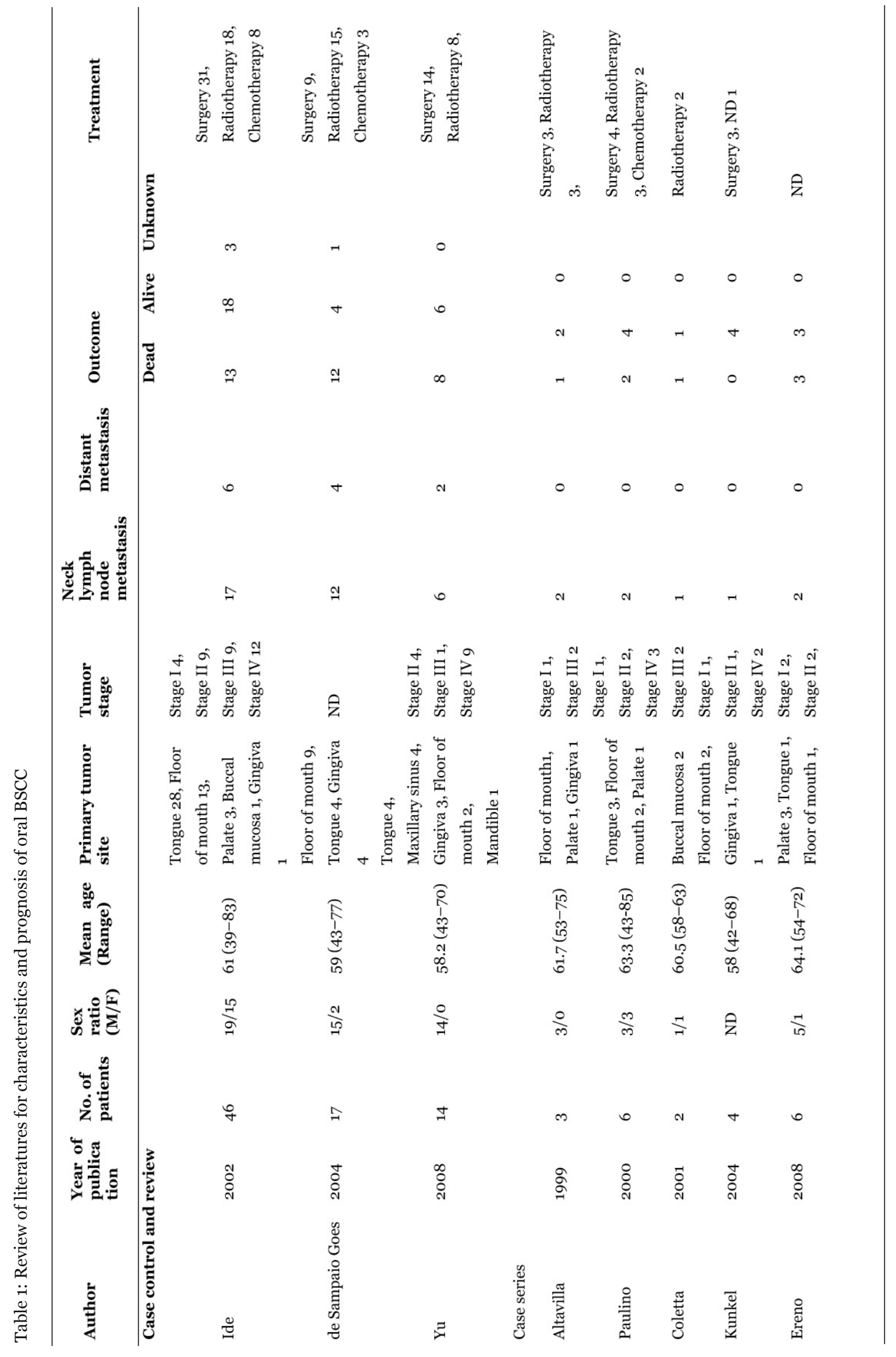


Table 2: Summary of clinical characteristics of oral BSCC

\begin{tabular}{|c|c|c|c|}
\hline Characteristics & & No. of patients & (\%) \\
\hline \multirow[t]{2}{*}{ Gender $(n=91)$} & Male & 66 & $(72.5)$ \\
\hline & Female & 25 & $(27.5)$ \\
\hline \multirow[t]{7}{*}{ Primary site $(n=107)$} & Tongue & 42 & $(39.3)$ \\
\hline & Floor of mouth & 32 & $(29.9)$ \\
\hline & Gingiva & 14 & $(13.1)$ \\
\hline & Palate & 10 & $(9.3)$ \\
\hline & Buccal mucosa & 4 & $(3.7)$ \\
\hline & Maxillary sinus & 4 & $(3.7)$ \\
\hline & Mandible & 1 & $(0.9)$ \\
\hline \multirow[t]{4}{*}{ Tumor stage $(\mathrm{n}=78)$} & Stage I & 12 & $(15 \cdot 4)$ \\
\hline & Stage II & 20 & $(25.6)$ \\
\hline & Stage III & 16 & $(20.5)$ \\
\hline & Stage IV & 30 & $(38.5)$ \\
\hline \multirow[t]{2}{*}{ Metastasis (n=95) } & Local nodal metastasis & 44 & $(46.3)$ \\
\hline & Distant metastasis & 13 & $(13 \cdot 7)$ \\
\hline \multirow[t]{3}{*}{ Outcome $(\mathrm{n}=95)$} & Dead & 41 & $(43.2)$ \\
\hline & Alive & 50 & $(52.6)$ \\
\hline & Unknown & 4 & $(4.2)$ \\
\hline \multirow[t]{3}{*}{ Treatment $(n=88)$} & Surgery & 71 & \\
\hline & Radiotherapy & 57 & \\
\hline & Chemotherapy & 16 & \\
\hline
\end{tabular}

of BSCC the primary treatment modality was surgery, with or without adjuvant radiotherapy [19]. In Soriano's series, the survival rates differed significantly between the case and control groups despite similar treatment modalities [3]. Survival rates for $\mathrm{T} 1$ and $\mathrm{T} 2$ cases were $64 \%$ for BSCC, and $86 \%$ for SCC. Similarly, the survival rates for $\mathrm{T}_{3}$ and $\mathrm{T}_{4}$ cases were $57 \%$ for $\mathrm{BSCC}$, and $85 \%$, for SCC. On the other hand, in a homogenous series of node-positive patients treated with chemoradiotherapy with or without neck dissection, the local control rates were similar. These data may suggest that BSCC has relatively good radiosensitivity in spite of its high rate of metastasis and poor survival rates [19].

The comparative analysis of the clinical course and prognoses of BSCC and conventional SCC has aroused controversy in literature. Although some authors have observed that BSCC is more aggressive than SCC, others suggest that the two have similar prognoses $[2,3,5]$. It is difficult to compare the biological features and clinical course of oral BSCC and conventional oral SCC in groups of matched patients. Three case-control studies comparing clinical and prognostic features in patients with BSCC included poorly differentiated and moderately to well-differentiated SCC of the oral cavity $[4,8,20]$. Yu et al. reported that the rate of cervical lymph node metastasis of BSCC was as high as $67 \%$, and that the rate of distant metastasis was $13 \%$ [4]. Radical surgeries were performed in all patients in that study; the tumor recurrence rate was $33 \%$, and the 5-year survival rate was $32 \%$. The researchers concluded that the biological behavior and prognosis of BSCC was similar to that of poorly differentiated SCC. Another case-control study reported a 5-year survival rate of $46 \%$ for oral BSCC $[8,20]$, and concluded that prognoses did not differ between patients with BSCC and those with conventional SCC. These studies support the idea that oral BSCC has a similar, rather than worse, behavior and prognosis in comparison to conventional SCC.

A summary of clinical characteristics previously reported for oral BSCC cases is presented in Tables 1 and 2. Clinical stages were classified as stage I for 12 patients (15.4\%), stage II for 20 (25.6\%), stage III for 16 
(20.5\%), and stage IV for 30 (38.5\%); thus, $59.0 \%$ of the reported cases presented at an advanced stage (stage III and stage IV). The incidence rate of local nodal metastasis was $46.3 \%$, and that of distant metastasis was $13.7 \%$. Patients were treated with surgery (71), radiotherapy (57), or chemotherapy (16) performed alone or combined with other therapies. The outcome for $43.2 \%$ of these patients was death; $52.6 \%$ patients survived and the survival was not reported for $4.2 \%$. Although almost $60 \%$ of the patients presented with oral BSCC at an advanced clinical stage, metastasis was controlled well in $52.6 \%$, local nodal metastasis was seen in $46.3 \%$, and distant metastasis was seen in $13.7 \%$. These results agree with the prognosis results reported by Yu et al. that BSCC in the oral cavity does not carry a worse prognosis than conventional SCC [4].

A more thorough work-up should be advocated for patients with BSCC than for patients with SCC [3, 7]. One study reported a 5-year actuarial distant metastasis rate of $29 \%$ for a head and neck BSCC cohort; the most common site of metastasis was the lung [7]. Another study reported a distant metastasis rate of $41 \%$ for BSCC; therefore, the authors recommended routinely performing a chest CT scan and FDG-PET to rule out early distant metastasis, and to include adjuvant chemotherapy in the treatment protocol [3]. A comparison of the course and prognosis of head and neck BSCC or SCC found that the survival rate was significantly higher for patients with SCC than for patients with BSCC. The rate of distant metastasis was six times higher in cases of BSCC [3]. Our summary of oral BSCC case reports showed an incidence of distant metastasis of $13.7 \%$, which is less than has been reported for head and neck BSCC cases as a whole (Tables 1 and 2). Chemotherapy has been recommended as an adjuvant to any treatment protocol for BSCC [19]. Patients should be assessed for oligometastases during follow-up to provide the best opportunity for treatment. Oligometastases, which occur frequently with BSCC, were found to be salvageable with wedge resection or radiotherapy, suggesting that early detection is important $[7,19]$.

\section{CONCLUSION}

Oral BSCC has a lower rate of distant metastasis than head and neck BSCC. In contrast to the aggressive behavior of BSCC occurring in the head and neck, BSCC occurring in the oral cavity appears to have a prognosis almost similar to that of conventional SCC. But sometimes, oral BSCC is more aggressive than conventional SCC. Therefore, when it comes to the follow-up of patients for metastases, a more thorough work-up is recommended for patients with oral BSCC than for patients with SCC.

$$
* * * * * * * * *
$$

\section{Author Contributions}

Kenji Yamagata - Substantial contributions to conception and design, Acquisition of data, Analysis and interpretation of data, Final approval of the version to be published

Rei Karube - Substantial contributions to conception and design, Acquisition of data, Analysis and interpretation of data, Final approval of the version to be published

Toru Yanagawa - Drafting the article, Revising it critically for important intellectual content, Final approval of the version to be published

Wolfgang Zemann - Drafting the article, Revising it critically for important intellectual content, Final approval of the version to be published

Philipp Metzler - Drafting the article, Revising it critically for important intellectual content, Final approval of the version to be published

Kojiro Onizawa - Drafting the article, Revising it critically for important intellectual content, Final approval of the version to be published

Hiroki Bukawa - Drafting the article, Revising it critically for important intellectual content, Final approval of the version to be published

\section{Guarantor}

The corresponding author is the guarantor of submission.

\section{Conflict of Interest}

Authors declare no conflict of interest.

\section{Copyright}

(C) Kenji Yamagata et al. 2013; This article is distributed under the terms of Creative Commons Attribution 3.0 License which permits unrestricted use, distribution and reproduction in any means provided the original authors and original publisher are properly credited. (Please see www.ijcasereportsandimages.com/copyright-policy.php for more information.)

\section{REFERENCES}

1. Wain SL, Kier R, Vollmer RT, Bossen EH. Basaloidsquamous carcinoma of the tongue, hypopharynx, and larynx: report of 10 cases. Hum Pathol 1986;17(11):1158-66.

2. Paulino AF, Singh B, Shah JP, Huvos AG. Basaloid squamous cell carcinoma of the head and neck. Laryngoscope 2000;110(9):1479-82.

3. Soriano E, Faure C, Lantuejoul S, et al. Course and prognosis of basaloid squamous cell carcinoma of the head and neck: a case-control study of 62 patients. Eur J Cancer 2008;44(2):244-50.

4. Yu GY, Gao Y, Peng X, Chen Y, Zhao FY, Wu MJ. A clinicopathologic study on basaloid squamous cell carcinoma in the oral and maxillofacial region. Int $\mathrm{J}$ Oral Maxillofac Surg 2008;37(11):1003-8.

5. Ereno C, Gaafar A, Garmendia M, Etxezarraga C, Bilbao FJ, Lopez JI. Basaloid squamous cell carcinoma of the head and neck: a clinicopathological and follow-up study of 40 cases and review of the literature. Head Neck Pathol 2008;2(2):83-91. 
6. Zbaren P, Nuyens M, Stauffer E. Basaloid squamous cell carcinoma of the head and neck. Curr Opin Otolaryngol Head Neck Surg 2004;12(2):116-21.

7. Thariat J, Ahamad A, El-Naggar AK, et al. Outcomes after radiotherapy for basaloid squamous cell carcinoma of the head and neck: a case-control study. Cancer 2008;112(12):2698-709.

8. de Sampaio Goes FC, Oliveira DT, Dorta RG, Nishimoto IN, Landman G, Kowalski LP. Prognoses of oral basaloid squamous cell carcinoma and squamous cell carcinoma: a comparison. Arch Otolaryngol Head Neck Surg 2004;130(1):83-6.

9. Kunkel M, Helisch A, Reichert TE, Bartenstein P, Wagner W. Surveillance of basaloid oral squamous cell carcinoma: the value of [18F]FDG-PET. Oral Oncol 2004;40(1):56-2.

10. Altavilla G, Mannara GM, Rinaldo A, Ferlito A. Basaloid squamous cell carcinoma of oral cavity and oropharynx. ORL J Otorhinolaryngol Relat Spec 1999;61(3):169-73.

11. Coletta RD, Cotrim P, Vargas PA, et al. Basaloid squamous carcinoma of the oral cavity: report of 2 cases and study of AgNOR, PCNA, p53, and MMP expression. Oral Surg Oral Med Oral Pathol Oral Radiol Endod 2001;91(5):563-9.

12. Hirai E, Yamamoto K, Yamamoto N, et al. Basaloid squamous cell carcinoma of the mandible: report of two cases. Oral Surg Oral Med Oral Pathol Oral Radiol Endod 2009;108(5):e54-8.

13. Ide F, Shimoyama T, Horie N, Kusama K. Basaloid squamous cell carcinoma of the oral mucosa: a new case and review of 45 cases in the literature. Oral Oncol 2002;38(1):120-4.

14. Coletta RD, Cotrim P, Almeida OP, Alves VA, Wakamatsu A, Vargas PA. Basaloid squamous carcinoma of oral cavity: a histologic and immunohistochemical study. Oral Oncol 2002;38(7):723-9.

15. Karatzanis AD, Fragkiadakis GM, Prokopakis EP, Koutsopoulos AV, Helidonis ES, Velegrakis GA. Basaloid squamous cell carcinoma of the soft palate: case report. Auris Nasus Larynx 2008;35(4):592-6.

16. Campos MS, Modolo F, de Oliveira JS, Pinto-Junior DS, de Sousa SC. Atypical presentation of oral basaloid squamous cell carcinoma. J Contemp Dent Pract 2009;10(2):98-104.

17. Joshi D, Shivkumar VB, Sharma SM, Gangane N. Cytomorphologic diagnosis of basaloid squamous cell carcinoma: a case report. Acta Cytol 2009;53(1):89-2.

18. Subramanian B, Agrawal K, Panda K. Basaloid squamous carcinoma of mandible. J Craniofac Surg. 2009;20(1):151-3.

19. Thariat J, Badoual C, Faure C, Butori C, Marcy PY, Righini CA. Basaloid squamous cell carcinoma of the head and neck: role of HPV and implication in treatment and prognosis. J Clin Pathol 2010;63(10):857-66.

20. Sampaio-Goes FC, Oliveira DT, Dorta RG, et al. Expression of PCNA, p53, Bax, and Bcl-X in oral poorly differentiated and basaloid squamous cell carcinoma: relationships with prognosis. Head Neck 2005;27(11):982-9.
Access full text article on other devices

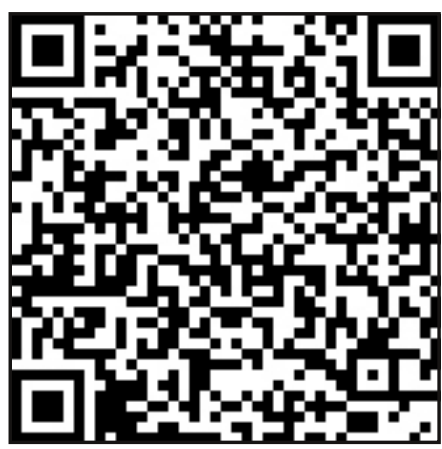

Access PDF of article on other devices

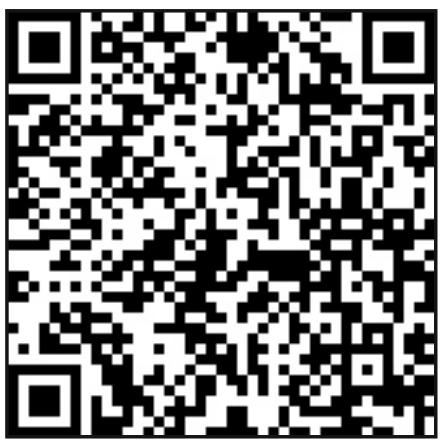

